Journal of Engineering and Applied Sciences 14 (Special Issue 8): 10271-10277, 2019

ISSN: 1816-949X

(C) Medwell Journals, 2019

\title{
Wear Resistance of Composite Materials Reinforced by Glass Fiber
}

\author{
${ }^{1}$ Mustafa Ahmed Rajab, ${ }^{2}$ Ziad T. Khodair and ${ }^{3}$ Asaad Hasan Lafta \\ ${ }^{1}$ Department of Mechanical, Technical Institute of Baqubah, Middle Technical University, \\ Baghdad, Iraq \\ ${ }^{2}$ Faculty of Science, University of Diyala, Baqubah, Iraq \\ ${ }^{3}$ Department of Production Engineering and Metallurgy, University of Technology, \\ Baghdad, Iraq
}

\begin{abstract}
The rate of wear is calculated from the weight data of the wear debris and the ratio of wear to the total content of the reinforcement materials. The amount of material removed from the composite material is obtained, due to the relative movement of the material with the steel disc. The loss of lost weight from composite materials means a decrease in the rates of wear. For example, increasing the wear rate of the composite material not reinforced by the other reinforced, accompanied by a large increase in plastic deformation and break the sub-surface layers. The randomly distributed particles and carbon fibers act as impediments to the deformation of the substrate (epoxy+resole resin) during loading.
\end{abstract}

Key words: Relative movement, composite materials, impediments, distributed, plastic deformation, carbon fibers

\section{INTRODUCTION}

The main functions of the fiber in a composite are to carry most of the load applied to composite and provide stiffness. For this reason, fiber materials which have high tensile strength and a high elastic modulus are often used for the fiber in composite (Rajab et al., 2017a-c). Carbon Fibers (CF) appeared in the market in 1960 and are produced from organic fibers (rayon, acrylics, etc.) or from remaining of petroleum or tar distillation (Anonymous, 2013). Carbon fibers are the strongest and stiffest reinforcing fibers for polymer composites, these fibers are the most commonly used after glass fibers. Carbon fibers can give galvanic corrosion in contact with metals. They're generally used together with epoxy, phenols, polyester where high stiffness and strength are required, i.e., space and automotive applications (Rajab et al., 2018). Another mechanical property that may be important to consider is the hardness, it is a measure of a material's resistance to localized plastic deformation. Shore hardness is measured with instrument known as a durometer and is also named durometer hardness (Rubab et al., 2014) (Fig. 1).

In the current study wear test, this method was adopted because it is easy method and can be inference the wear rate because it gives the amount of wear debris. This method is summarized by weighted of the sample before and after the test and the difference between the two weights represents the amount of wear debris (Conner, 2001; Rajab and Hameed, 2017). Carbon has two natural crystalline allotropic forms: graphite and diamond. Graphite derives its name from the Greek word "graphein" (Rajab and Hameed, 2017). Graphite is defined as an allotropic form of the element carbon consisting of layers of hexagonally arranged carbon atoms in a planar condensed ring system (grapheme layers, these planes also called basal plane). The layers are packed parallel to each other in a three dimensional structure. The atoms within the rings are bonded covantly, whilst the layers are loosely bonded together by Van der Waals forces (Maitra et al., 2009). The high degree of anisotropy in graphite results from the two types of bonding acting in different crystallographic directions. For example, graphite's ability to from a solid film lubricant comes from these two contracting chemical bonds. The fact that weak Van der Waals forces govern the bonding between individual layers permits the layers to slide over one another making it an ideal lubricant. Graphite is generally, grayish-black, opaque and has a lustrous black sheen. It is unique in that it has properties of a metal and a non-metal. It's flexible but not elastic has a high thermal and electrical conductivity and is highly refractory and chemically inert (Rajab et al., 2017a-c). Silica occurs commonly in nature as sand stone, silica sand or quartzite. It is the starting material for the production of silicate glasses and ceramics. Silica is one of the most abundant oxide materials in the Earth's crust. It can exist in an amorphous form (vitreous silica) or in a variety of crystalline forms. Often it occurs as a non-crystalline oxidation product on the surface of silicon or silicon

Corresponding Author: Mustafa Ahmed Rajab, Department of Mechanical, Technical Institute of Baqubah, Middle Technical University, Baghdad, Iraq 

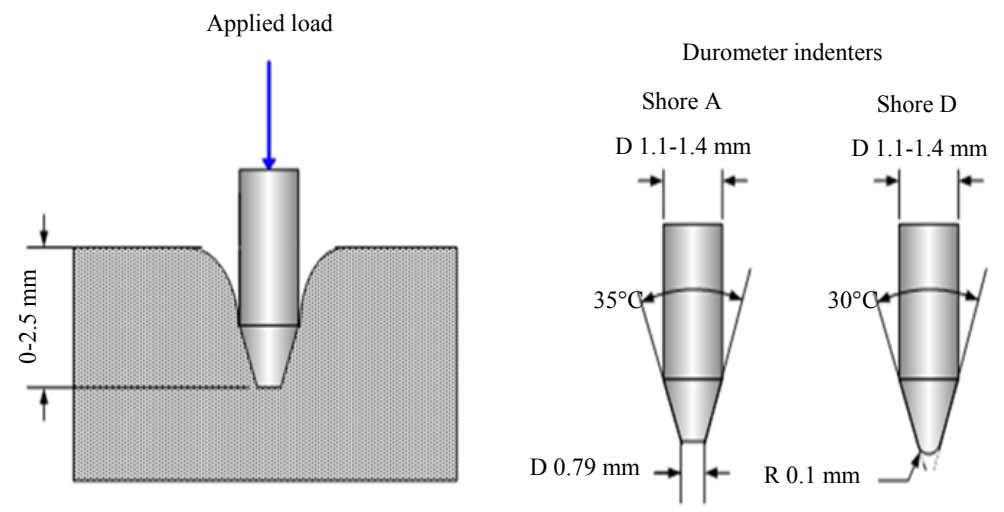

Fig. 1: Durometer indenters (Rubab et al., 2014); Durometer hardness test

compounds (Mallick, 2007; Rajab et al., 2017a-c). The mechanical strength of polymers can be improved by reinforcing the polymer matrix by the addition of filler materials like ( silica, alumina, zirconia). These composite materials are stronger than the bulk (un-reinforced) polymers as a result of the high-strength reinforcement phase obliging the applied loads (Berthelot et al., 1999). Composite materials in this regard represent nothing but a giant step in the ever-constant endeavor of optimization in materials. Strictly speaking, the idea of composite materials is not a new or recent one. Nature is full of examples where in the idea of composite materials is used (Rajab et al., 2017a-c). Phenol formaldehyde resins is a type of polymer is made from two main substances: phenol and formaldehyde. Phenol is a colorless solid compound but when exposure to oxidation in the air its colored by pink Then brown. It has strong odor and penetrating. Phenol is widely used in the manufacture of materials for plastics including drinking water bottles as well as in the clothing industry. Phenol is used in the installation of nylon. It has medical uses, it is used in the manufacture of disinfectants, lotions, ointments, topical anesthetics and the pharmaceutical industry. The water solution which contains a concentration of $40 \%$ formaldehyde is called formalin. This is used as a preservative for tissues and in embalming with a boiling point of $21^{\circ} \mathrm{C}$, it is used in veterinary and in dentistry as well as in the production of chemicals and polymers and is often used in the manufacture of coatings and explosives (Rubab et al., 2014). In general, phenol-formaldehyde produced by two ways for manufacture two types of polymers, namely novolac and resole. Novolac is a type of polymers produced by mixing formaldehyde ( $37 \%$ water solution) with phenol by added an acidic helper (sulfuric, phosphoric or oxalic acid) and heated to the required degree and then equivalent the reaction mixture and remove the water by distillation (in its final stages under discharge) to a temperature estimated $160^{\circ} \mathrm{C}$. Resole is a type is produced by added basic helper with more formaldehyde relative to phenol. Initially, its consists (Oligomer) is called a resole and it is not needed to a hardener (HMTA) but it need just heat treatment (Rajab et al., 2018; Conner, 2001). By (2013), Hamid S., studied the mechanical properties (tensile, bending and hardness) of unsaturated polyester resin reinforced with silica particles in different weight fractions $(10,20,30$ and $40 \%)$. Results showed decreasing in tensile strength and flexural strength with increasing particle concentration, increasing in hardness, tensile modulus and bending modulus with increasing in particle concentration. Vikram et al. studied the influence of different content ratios and lengths of carbon fiber reinforced epoxy composites. Carbon fiber is taken in the $3,5,7 \%$ weight in order to suspend on epoxy resin with different fiber lengths such as 1,2 and $3 \mathrm{~cm}$. Moreover, they studied the thermal properties such as TGA and DSC to investigate the influence of change in fiber length on carbon fiber-epoxy composites. Significant improvement in tensile and flexural strengths of carbon fiber-epoxy composites has been observed by the different fiber lengths. Flexural strength, flexural modulus, tensile strength and modulus were increased correspondingly up to $5 \% \mathrm{wt}$. and $2 \mathrm{~cm}$ length of carbon fiber reinforced epoxy and decreases with further addition of fiber contents, i.e., $7 \% \mathrm{wt}$. They concluded that carbon fiber-epoxy composites can be used for high strength, stiffness and bending applications in aerospace, automobile and marine and lightweight article applications. Overall studies indicated that the carbon fiber reinforced composites at $2 \mathrm{~cm}$ length of carbon fiber and $5 \% \mathrm{wt}$. loading are promising candidates for structural applications where high strength and stiffness is indispensable. In (2015), Jweeg et al. designed a new athletic prosthetic foot. The foot was manufactured by using epoxy reinforced by carbon fibers and that gives good mechanical response. The impact tester was designed and manufactured to perform the test. For the same dropped level, the impact response of the samples 
with glass fiber and carbon fiber have the same peak load for different drop angle but. In addition, it was clear that the responses of the sample manufactured with carbon fiber were more smoothness than the sample manufactured with the glass fiber. In (2016), Jagadale and Raut investigated the mechanical properties (tensile strength and shear strength) of glass fibers reinforced polymer matrix with different fibers volume fraction $(40,50$ and $60 \%)$, hand lay-up and compression molding were used to prepare the samples. Results showed better mechanical properties at volume fraction (50\%), further increase in the fiber content leads to increase in the mechanical properties but the composites start to delaminate.

\section{MATERIALS AND METHODS}

\section{Experimental procedure}

Materials used: The materials used in this research are: epoxy resin supplied by (Sikadur ${ }^{\circledR}-52$ Injection Type N), the base used to mix is mixed $3 \mathrm{~g}$ of resin with every $1 \mathrm{~g}$. of the hardener. Table 1 shows the properties of the epoxy resin.

Resole resin: Is consists of one part only. Resole resin was the first wholly synthetic polymer to be commercialized (Maitra et al., 2009). It has become one of the most widely utilized synthetic polymers, since, Baekeland developed a commercial manufacturing process by Rajab et al. (2017a-c). Resole is synthesized under basic conditions with the Formaldehyde $(\mathrm{F})$ and phenol (p) molar ( $\mathrm{F} / \mathrm{p}>1$ ) (Rajab and Hameed, 2017).

Glass fiber: The type of glass fiber used in this research as reinforced materials are woven E-glass fiber from the Tenax Company, England, made as reinforcement (Table 2).

The practical part includes the preparation of the raw materials and how to prepare them in addition to the mechanical tests carried out on the overlapping material. These materials were mixed with different weight. At

Table 1: The properties of epoxy resin used in this research Properties Values (MPa at $\left.25^{\circ} \mathrm{C}\right)$

Tensile strength

Flexural strength

Compressive strength

Density

Viscosity

40

63

55

1.5

439

Table 2: The properties of glass fiber used in this research Properties Values

Compressive strength $1077 \mathrm{MPa}$

Tensile strength $\quad 3521 \mathrm{MPa}$

Young modulus

Poisson's ratio

$69.8 \mathrm{GPa}$

0.23

Density

$2.6 \mathrm{gcm}^{-3}$ first, the substance was mixed with phenol formaldehyde resin, called resole. Different mixing ratios were used to obtain the samples. For the purpose of making samples for the necessary tests to obtain the mechanical properties and analysis and compare them with the mechanical properties of the alloy which is the part of the original which was chosen for the purpose of replacement of the alloy composite material used in the search for weight loss and improve mechanical properties. The oil tank was selected in the engine as part of the study and a simulation program was used as shown in Fig. 2 where the composite material used in the research is tested and compared to the alloy material from which the part was originally manufactured.

Material used: The material used in this research divided to two matrix material:

- Epoxy resin

- Phenol formaldehyde (resole) resin

Phenol-Formaldehyde $(\mathrm{PF})$ resole resin was the first wholly synthetic polymer to be commercialized (Anonymous, 2013). It has become one of the most widely utilized synthetic polymers, since, Baekeland developed a commercial manufacturing process in 1907 (Rajab et al., 2018). Resoles are synthesized under basic conditions with the Formaldehyde $(\mathrm{F})$ and Phenol $(\mathrm{P})$ molar ratio ( $\mathrm{F} / \mathrm{P}>1$ ) (Anonymous, 2013). Resole was curing by heated it (Fig. 2).

Technical testing procedures: There are several instruments and equipment utilized to measure and determine the mechanical properties of the materials under investigation.

Mechanical testing instruments: Mechanical properties were measured at room temperature $\left(20-30^{\circ} \mathrm{C}\right)$.

Hardness: The hardness is the ability of a material to resist penetration, the shore $\mathrm{D}$ hardness values showed that the results of the different mixing ratio (Fig. 3).

Wear test instrument: The pin-on-disk instrument is popular wear testing apparatus. This instrument consists

\begin{tabular}{|c|c|c|}
\hline Hardnes & $4 \mathrm{~mm} \underset{10 \mathrm{~mm}}{\stackrel{+}{\rightleftarrows}}=10 \mathrm{~mm}$ & ASTM-D2240 \\
\hline Wear & $10 \mathrm{~mm} \quad 20 \mathrm{~mm}$ & ASTM-G99 \\
\hline
\end{tabular}

Fig. 2: Sample dimensions and standard specifications of the testing specimens (Rajab et al., 2017a-c) 


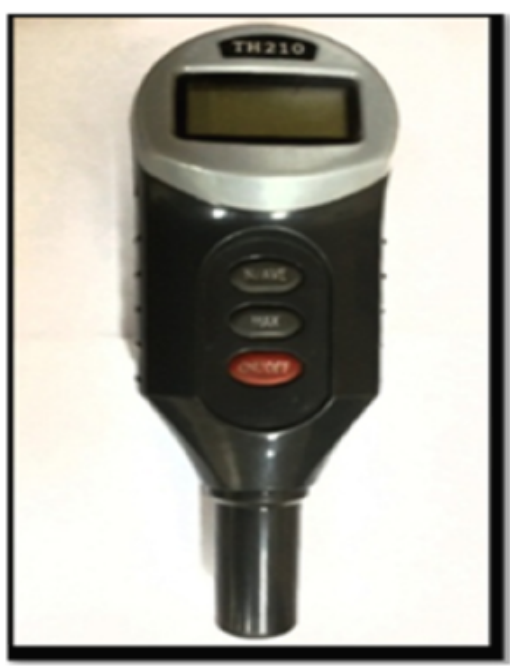

Fig. 3: Hardness test (shore D)

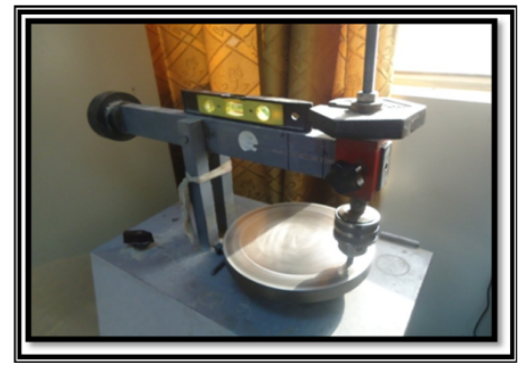

Fig. 4: Pin-on-disk

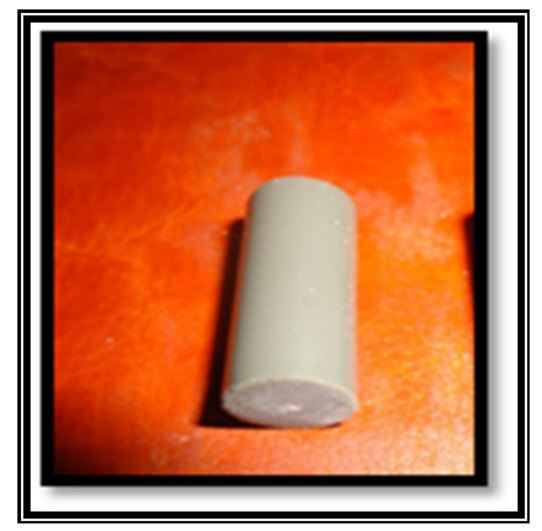

Fig. 5: Wear test sample

of electrical motor which rotates at angular velocity, the motion is transferred from the electrical motor to the disc. The disc has angular velocity of (720 rpm) (Fig. 4 and 5).

Scanning Electron Microscope (SEM): Scanning Electron Microscope (SEM) in Fig. 6, model Inspect S50, made in (Holland) used to investigate the morphology

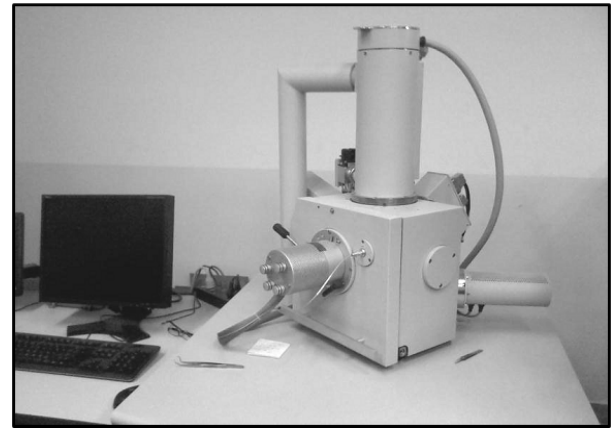

Fig. 6: Scanning electron microscope device

of the sample surface. The test was done in the Department of Applied Science at the University of Technology.

\section{RESULTS AND DISCUSSION}

Hardness test: It is known that most tests of hardness depend on the material resistance to penetrate at the outer surface and there are different ways to evaluate the hardness in the current research has been the hardness test using the hardness (shore durometer) type (shore D) and through the results note that the hardness values of samples are increasing with Increase the fractional fraction of the supported particles (graphite, silica), especially, if the particle size is small and the homogeneous distribution is distributed within the floor. The studies indicate that the hardness values reached their highest value when increasing the volume fracture to $15 \%$ and then decreased to their lowest values at the volumetric fraction $(20 \%)$. This is due to the high viscosity gained by the prepared material when adding high ratios of the particles of graphite into the base material which is in the liquid state which caused the difficulty of penetration of the base material into the interstitial fissure of the fibers and porous interstices within the composite material, resulting in creating a lot of gaps in the prepared material and despite attempts to get rid of gaps and aerobic space I kept some of them p. Since, the hardness of the surface of the material and the presence of particles of graphite and silica at the surface of the material and the properties of these particles of hardness, the resistance to the force exerted on them are few, so, the increase in the values of hardness Be few with increased particle fracture. The effect of particle sizes on hardness values is that hardness values increase with low particle size (graphite or silica) and this is due to the use of particles in small sizes which facilitates the process of penetration into the base material and into the fiber network interface and into the pores that which was made during the process of preparing the overlays, all of which helped to increase the area of contact between the 
components of the composite material prepared and then increase the interdependence between them and in an integrated which gave more positive values when examining the hardness and conversely when the presence of large particles will be a barrier either flow of material. And the fiber nature has a great role in determining the values of prayer because the hardness of those fibers vary according to the type of fiber. Because some of them are made of ceramic materials while other fibers are made of polymeric materials. The hardness test was performed by a regression method with four readings per sample. The most suitable method for measuring the hardness was the hardness values obtained from them which reflect the condition of the material as a whole and not just the surface state. It is mainly dependent on the amount of energy absorbed by it. Absorbs a greater amount of energy which leads to the bounce of the ball to a higher altitude than if the material is solid in which case will return to the rise of the largest result of absorption of less energy and the results obtained confirm this. Fiber orientation has an influential role in hardness values. The calcium-reinforced samples $\left(90-0^{\circ}\right)$ gave the highest values of hardness compared to random-pattern samples. This indicates that the use of standard-size glass fibers gives more positive results in the reinforcement process and it is noted that the lattice system in fiber reinforcement increases the hardness values of the samples with the total volume fracture and these hardness values increase with the number of reinforcing layers which confirms the positive effect of the arming process with this fiber (Maitra et al., 2009). The concept of hardness can be counted as a measure of the plastic deformation that the material can suffer under the influence of external stress. Thus, particle reinforcement (silica or graphite) increased the hardness of the material due to increased resistance to plastic deformation. It has been found that the increase in the reinforcement materials and then the glass fiber armament, led to an increase in the hardness of the material, this may be due to the fact that the hardness is a property of the surface, so, this behavior is expected to hardness (Fig. 7).

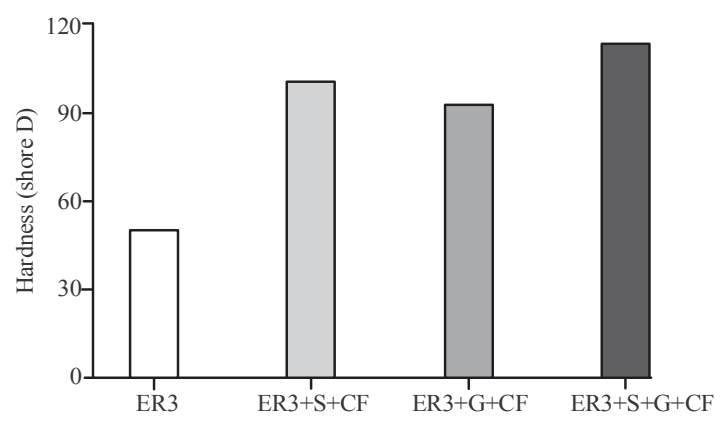

Fig. 7: Hardness testing using the hardness (shore durometer) type (shore D)
Previous studies by Rajab et al. (2017a-c) indicate that the increase in the percentage of reinforced materials also led to a decline in the hardness values of the prepared models due to a decrease in the ratio of the base material. This means a weakness in the cohesion of prepared compositions. However, the above figure indicates a rise in hardness values with increasing glass fiber ratio, especially, to $3 \%$ which confirms the positive effect of the process of strengthening this type of fiber because it possesses characteristic mechanical properties make it efficient to bear part of the load on the composite material. The figure shows the convergence between the hardness of the samples supported by silica or graphite and glass fiber reinforced.

The weight of the load was measured in the wear rate of the composite materials used in the research. Three loads $20,15,10$ Newton, respectively were adopted and 10 $\min$ for a hard drive $(266 \mathrm{HB})$. The results of the practical tests showed that the increase in the load increased lead to increase the rate of wear and tear due to increased friction strength and because the friction Force (F) Newton is proportional to the pressure force $(\mathrm{N})$ : $\mathrm{F} \alpha \mathrm{N}$ :

$$
\mathrm{F}=\mu^{*} \mathrm{~N}
$$

If $\mu$ the coefficient of friction is as well as high temperature between the sample surface and disk. The two surfaces are composed of protrusions and grooves and the beginning of contact between the two surfaces occurs at the sharp protrusions and under the impact of pregnancy, the stress is concentrated on the sharp protrusions which leads to a deformation of these stenosis and increase the pregnancy lead to increased deformation at the tops of protrusions and the region near. The surface is drilled as a result of the impact of the minutes resulting from the surface crust crash. The small cracks accumulate together, leading to the removal of the surface layers of the debris formed in the form of thin minutes. This increases the plastic deformation with the increase of the load. That the sliding surfaces at low loads leads to the formation of a protective surface membrane reduces the contact between the two surfaces and therefore, the force required to cut the bond between the protrusions less than the force required to cut the molecular bonding of the chains of the mixture and thus, less wear rate but increase the load is getting cracking surface membranes and adhesion is strong between the two surfaces and therefore, the force required to cut the connected protrusions is higher than the force required to cut the molecular bonding of the mixture chains, so, the wear rate increases. And the strength of friction between the two surfaces affect the rate of wear and tear by getting emotions as a result of the generation of pressure stress resulting from the shedding of pregnancy and these emotions cause the 
transfer of part of the surface of the sample contact the surface of the disk and therefore the contact area will increase continuously, leading to increased wear and tear (Rajab et al., 2018). Moreover, the temperature has an effect on the rate of wear and tear and the increase in temperature increases the softness of the material and this leads to increased adhesion between the protrusions of the two surfaces and thus, increase the rate of wear and tear. There may be a decrease in the rate of wear and tear as a result of the stress on the model works on the surface of sharp protrusions which reduces the rate of wear and tear but increase the burden of pregnancy leads to the formation of sharp bumps again and increase the rate of wear and tear (Rajab et al., 2017a-c). It is noted that all of the above-mentioned machineries have obtained the models used and the transition from wear transition to severe wear, indicating that wear and tear increased with increased load or transition from transition wear) to moderate wear (Mild wear) is due to the phenomenon of emotional dependence and the transition of wear and tear from one stage to another depends on the test conditions and chemical composition of the mixture. This is what the researcher (Hallel) found that the rate of wear and tear of pure epoxy increases with the increase of the load (Mallick, 2007) (Fig. 7).

Effect of alternative breast change: The results show that the increase in surface load increases the roughness of the surface as well as increase the depth of the effects of wear and tear at the speed of the slide $\left(3.1415 \mathrm{msec}^{-1}\right)$, the hardness of the iron disk $(266 \mathrm{HB})$ and the load of 10 Newton, low and the effects of wear and tear is not deep compared to the surface of the sample exposed to carry (15) Newton and increase surface roughness at the load (20) Newton and as shown in Fig. 8. This difference is due to the roughness of the surfaces and the depth of the effect that the plastic deformation occurring at the tops of the protrusions and the area near the surface increases with the increase of the load.

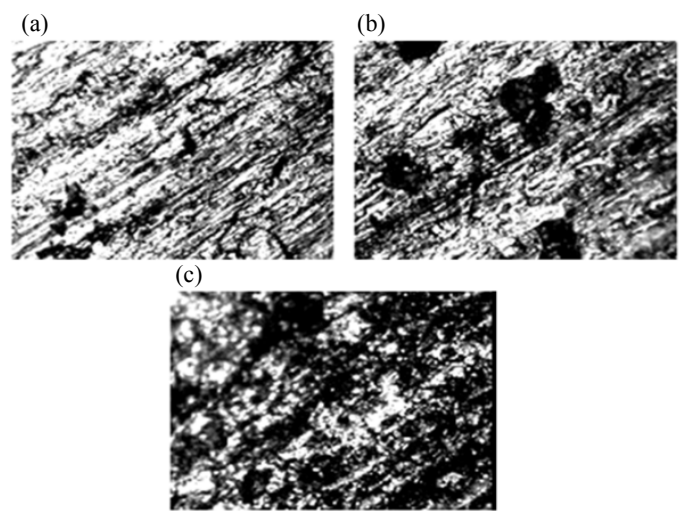

Fig. 8(a-c): The effect of change in load bearing with the effect of wear and tear on the surface of the sample. $500 \mathrm{X}$
Second; effect of immersion solutions in the rate of wear: The results of the imaging, under the load of 10 Newton, the hardness of the iron disk (266 HB) and the speed of the slide $\left(3.1415 \mathrm{msec}^{-1}\right)$ show that the surface of the sample is smooth in normal conditions (Fig. 9a) and is more rough after immersion in chemical solutions due to increased wear and tear after immersion, In base solution (Fig. 9c), the surface is coarser than in the acid solution (Fig. b) because of the effect of the base solution on increasing the wear rate more than the acid solution (Fig. 9).

Effect of sliding speed in wear rate: The velocity of slippage was measured in the wear rate of the polymer mixture. The adopted slippage velocity was 4.1887 , $3.1415,2.0943 \mathrm{msec}^{-1}$, respectively, the test time was $10 \mathrm{~min}$ and the copper and iron test chips were found. Sliding velocity this behavior of the rate of wear and tear is observed for both dry conditions and in the case of immersion in chemical solutions and test chips (iron and copper).

The behavior of the rate of wear and tear in normal conditions: Previous studies have found that there are two areas of slippery speed, namely the low slippery speed zone $\left(2.0943,3.1415 \mathrm{msec}^{-1}\right)$ and the high slip speed zone $\left(4.1887,2.0943 \mathrm{msec}^{-1}\right)$. In the slippery speed zone as a result, the sloping velocity consists of a layer of surface membrane that reduces the friction of the two surfaces. Therefore, the surface temperature is not effective to distort the bumps of the two surfaces. Therefore, the wear rate is inversely proportional to the sliding speed (Rubab et al., 2014). The second reason is the presence of wear and tear minutes in the region by reducing the rate of wear and tear. It occurs that at low speeds, the minutes of wear and tear are combined with the sliding sample. This causes the surface of the disc to be enlarged due to filling gaps and reducing the bumps (Rajab et al., 2017a-c). As for the high speed, it is noted

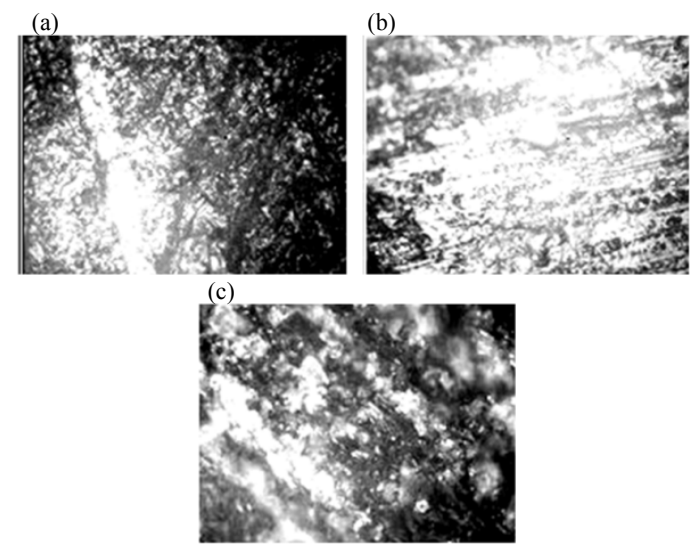

Fig. 9(a-c): The effect of immersion in chemical solutions with the effect of wear and tear on the surface of the sample. $500 \mathrm{X}$ 


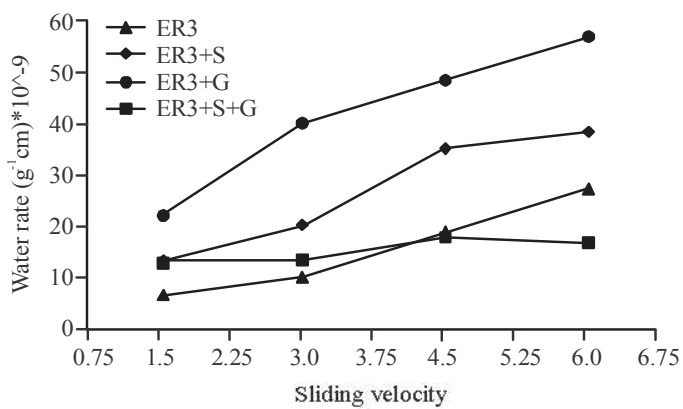

Fig. 10: The behavior of the rate of wear and tear in normal conditions

that the rate of wear and tear increases with the increase of the slippery speed. This is due to the fact that the high temperature increases the deformation of the contact bumps and the temperature softens the surface of the sample with the rotor disc, thus, facilitating the separation of wear minutes (Rajab and Hameed, 2017). The other reason is that at the high speed, the centrifugal force removes the wear and tear minutes from the sliding sample on the turntable, thus, increasing wear and tear with increasing sliding speed. These reasons were observed for the sliding (natural) patterns on the test disc (iron and copper). This finding is consistent with Lhymn's study of the emergence of two slippery velocity zones when testing wear-and-tear for carbon fiber-supported models (Rajab et al., 2017a-c) (Fig. 10).

\section{CONCLUSION}

The rate of wear and tear in the dry state is less than in the case of immersion in chemical solutions and for the same reasons mentioned earlier. Note that the rate of wear and tear when immersed in chemical solutions was the effect of acid solution less than in the base solution. The rate of wear and tear of the iron disc is less than the wear rate of the copper disc and for the same reasons described previously.

\section{REFERENCES}

Anonymous, 2013. Effect of resin molecular architecture on epoxy thermoset mechanical properties. Materials Design Inc., San Diego, California.

Berthelot, J.M. and J.M. Cole and F.F. Ling, 1999. Composite Materials: Mechanical Behavior and Structural Analysis. Spriger-Verlag Inc., New York.
Conner, A.H., 2001. Wood: Adhesives. In: Encyclopaedia of Materials: Science and Technology, Buschow, K.H.J. (Ed.). Elsevier Science Ltd., New York, USA., ISBN-13: 9780080431529, pp: 9583-9599.

Maitra, U., K.E. Prasad, U. Ramamurty and C.N.R. Rao, 2009. Mechanical properties of nanodiamondreinforced polymer-matrix composites. Solid State Commun., 149: 1693-1697.

Mallick, P.K., 2007. Fiber-Reinforced Composites: Materials, Manufacturing and Design. 3rdEdn., CRC Press, Boca Raton, Florida, Pages: 620.

Rajab, D.M.A. and A.H.I. Hameed, 2017. Hardness and wear resistance of composite materials reinforced by silica, graphite and carbon fiber. Proceedings of the International Congress of the Water Management, Energy, Food and Agricultural Techniques, November 7-10, 2017, Istanbul Turkey, pp: 7-10.

Rajab, M.A., A.A. Hammod, E.I. Kader and A.H.I. Hameed, 2018. Mechanical properties (Tensile, Hardness and Shock Resistance) for the phenol formaldehyde resin with Epoxy resin. Diyala J. Eng. Sci., 4: 55-60.

Rajab, M.A., E.I. Kader and A.H.I. Hameed, 2017b. Flexural and shock strength of silica, graphite and carbon fiber reinforced composite materials. Proceedings of the 5th International Conference on Innovation Challenges in Multidisciplinary Research Practices (ICMRP), December 15-16, 2017, Singapore, Asia, pp: 257-267.

Rajab, M.A., E.I. Kader and A.H.I. Hameed, 2017a. Study the mechanical behavior of advanced composite materials. Proceedings of the 2nd International Scientific Conference on Faculty of Bilad Rafidain-University, April 18-19, 2017, Alrafidain University College, Baghdad, Iraq, pp: 18-19.

Rajab, M.A., E.I. Kader, A.A. Hamod and A.H.I. Hameed, 2017c. Mechanical properties of silica, graphite and carbon fiber reinforced composites. Intl. J. Eng. Technol., 9: 3532-3535.

Rubab, Z., A. Afzal, H.M. Siddiqi and S. Saeed, 2014. Augmenting thermal and mechanical properties of epoxy thermosets: The role of thermally-treated versus surface-modified $\mathrm{TiO}_{2}$ nanoparticles. Mater. Express, 4: 54-64. 